\section{(2) OPEN ACCESS}

\title{
Ergothioneine is associated with reduced mortality and decreased risk of cardiovascular disease
}

\author{
Einar Smith 당 , Filip Ottosson, Sophie Hellstrand, Ulrika Ericson, \\ Marju Orho-Melander, Céline Fernandez, Olle Melander
}

\begin{abstract}
- Additional material is published online only. To view, please visit the journal online (http://dx.doi.org/10.1136/ heartjnl-2019-315485).
\end{abstract}

Department of Clinical Sciences, Lund University, Malmö, Sweden

\section{Correspondence to}

Mr Einar Smith, Department of Clinical Sciences, Lund University, Malmö 21428, Sweden; einar.smith@med.lu.se

CF and $\mathrm{OM}$ are joint senior authors.

Received 29 May 2019 Revised 19 September 2019 Accepted 1 October 2019 Published Online First 31 October 2019

\section{ABSTRACT}

Objectives We recently identified a health conscious food pattern (HCFP) associated with reduced risk of cardiometabolic disease. However, the molecular events linking the healthy food pattern to reduced risk of cardiometabolic disease are unknown. Our aim was to identify plasma metabolites associated with the HCFP and test if such metabolites predict cardiometabolic disease and mortality.

Methods Using liquid-chromatography massspectrometry, 112 plasma metabolites were measured in 3236 participants without cardiovascular disease (CVD) and diabetes mellitus from the population-based Malmö Diet and Cancer study. Metabolites associated with the HCFP were identified using multivariable adjusted linear regressions followed by Bonferroni correction. The healthy dietary biomarkers were subsequently related to risk of cardiometabolic disease and mortality during long-term follow-up with multivariable adjusted Cox proportional hazards models.

Results During a median follow-up time of 21.4 years, 603 participants developed CVD, 362 developed diabetes mellitus and 843 participants died. Five healthy dietary biomarkers were associated with the HCFP at baseline $(p<0.0004)$ and four predicted at least one of the studied end points $(p<0.05)$. Ergothioneine was the metabolite most strongly connected to the HCFP and was associated with a lower risk of coronary disease (HR per $1 \mathrm{SD}$ increment of ergothioneine, $\mathrm{HR}=0.85, \mathrm{p}=0.01$ ), cardiovascular mortality $(H R=0.79, p=0.002)$ and overall mortality $(H R=0.86, p=4 e-5)$.

Conclusions We identified that higher ergothioneine was an independent marker of lower risk of cardiometabolic disease and mortality, which potentially can be induced by a specific healthy dietary intake.

\section{INTRODUCTION}

Dietary interventions have been suggested as a tool to improve cardiometabolic health to decrease the risk for type 2 diabetes, coronary artery disease (CAD), stroke and mortality. ${ }^{12}$ Randomised dietary intervention studies with hard outcomes are, however, challenging and there is controversy as to which dietary qualities would promote cardiometabolic health the most ${ }^{3}$ even though several 'healthy/ prudent' data-driven dietary patterns have been found to associate with lower risk of cardiometabolic disease in observational prospective studies. ${ }^{4}$ Understanding the molecular events resulting from dietary intakes, and their relationship to disease and health outcomes would facilitate future intervention studies through identification of diet-modifiable metabolic pathways and disease mechanisms.

Metabolomics can be used to measure how the levels of circulating metabolites are connected to dietary intakes. A large number of metabolites in the human circulation derive directly from the digestion and metabolism of food components and constitute the food metabolome. ${ }^{5}$ Both endogenous plasma metabolites and metabolites from the food metabolome have been shown to correlate with certain dietary patterns estimated using questionnaires on dietary intake in cohort studies, ${ }^{6}$ and with certain food items in randomised controlled feeding trials. ${ }^{7}$ The connection between diet and the metabolome is particularly interesting because circulating levels of specific metabolites have previously been shown to predict future risk of type 2 diabetes, ${ }^{8} \mathrm{CAD}^{9}$ and premature mortality. ${ }^{10}$ By combining data from dietary intake, metabolomics and clinical outcomes, certain metabolites could be identified as associated with both a healthy diet and cardiometabolic health, thus pinpointing potential mechanisms of action of diet at the molecular level and candidate substances for future interventions by randomised controlled trials aiming to improve cardiometabolic health. ${ }^{11}$

Here, we measured fasting plasma levels of 112 metabolites from the baseline examination of a Swedish population-based prospective cohort study, the Malmö Diet and Cancer study, comprising 3236 individuals without cardiovascular disease (CVD) and type 2 diabetes at study entry. We first assessed these metabolites' association with a previously identified health conscious food pattern (HCFP) which was associated with protection from CVD and type 2 diabetes. $^{12}$ Thereafter, HCFP-associated metabolites were related to incidence of type 2 diabetes, CVD, cardiovascular and overall mortality during a median follow-up time of 21 years. Our aim was to identify metabolites connected to both the HCFP and to protection from cardiometabolic disease in order to highlight metabolic pathways and mechanisms linking healthy dietary intake to cardiometabolic health.

\section{METHODS}

\section{Study population}

The Malmö Diet Cancer (MDC) study is a population-based prospective cohort study consisting of 28098 individuals who attended a baseline examination between 1991 and 1996 in Malmö, Sweden. ${ }^{13}$ From this cohort, 6103 people
To cite: Smith $\mathrm{E}$, Ottosson $\mathrm{F}$ Hellstrand $\mathrm{S}$, et al. Heart 2020;106:691-697. 
were randomly selected to participate in the cardiovascular cohort (MDC-CC), a cohort designed to study the epidemiology of carotid artery disease. ${ }^{14}$ All the participants in MDC-CC underwent a medical history and physical examination, laboratory assessment as well as a comprehensive dietary assessment and filled out a lifestyle questionnaire.

All participants provided written informed consent. This research was done without patient involvement.

A random sample of 3833 MDC-CC participants was included for metabolite measurement as described below in the section "Metabolite measurements". Participants with prevalent CAD $(n=80)$, stroke $(n=26)$ and/or type 2 diabetes $(n=348)$ at baseline, as well as those with incomplete data on covariates $(n=43)$ or dietary intake $(n=120)$ were excluded from all analysis. The remaining 3236 participants constituted our study sample. Out of these participants, 2513 had information on adherence to the previously derived HCFP, and constituted the study sample when circulating metabolites were related to the HCFP.

\section{Dietary data}

The MDC study used a diet history method that combined a 7-day-menu book, a 168-item food frequency questionnaire and a 45 min interview. The MDC method is described in detail both elsewhere and in the (online supplementary file 1). ${ }^{15}$ In order to define a healthy dietary intake, we used the previously published HCFP derived from the MDC cohort by principal components analysis of participants' food intakes. ${ }^{12}$ The HCFP was characterised by high intake of fibre-rich bread, breakfast cereals, fruits, vegetables, fish and low-fat yoghurt and by low intake of low-fibre bread.

\section{Baseline data collection}

Data on baseline smoking status and use of antihypertensive treatment were obtained from the baseline questionnaire. BMI was calculated as weight in kilograms divided by the square of the height in metres. Systolic and diastolic blood pressure were measured after $10 \mathrm{~min}$ of rest in the supine position. Leisure time physical activity was assessed by asking the participants to estimate the number of minutes per week they spent on 17 different activities. The duration was multiplied with an activity-specific intensity coefficient and an overall leisure time physical activity score was created.

Laboratory analyses were conducted on overnight fasted blood samples obtained at baseline examination. Analyses of plasma lipids and whole blood glucose were performed according to standard procedures at the Department of Clinical Chemistry, Skåne University Hospital in Malmö, which is linked to a national accreditation control system. The levels of lowdensity lipoprotein (LDL) cholesterol were calculated using the Friedewald formula.

\section{End point definition}

The end point definition is further explained in the supplementary material. Briefly, end points were retrieved by linking the 10-digit Swedish personal identification number with previously used and validated registers. CAD was defined as coronary artery revascularisation, fatal or non-fatal myocardial infarction or death due to ischaemic heart disease.

\section{Metabolite measurements}

Profiling of plasma metabolites was performed using LC-MS and has previously been described in details ${ }^{16}$ and is further explained in the supplementary material. Measured metabolites are listed in the in-house metabolite library (online supplementary table 1).

\section{Statistical analyses}

$\mathrm{R}$ (V.3.5.1) was used for all statistical analyses. Due to nonnormality, metabolite data were log-transformed and scaled to multiples of $1 \mathrm{SD}$ and centred on zero prior to statistical analysis.

The associations between baseline fasting levels of circulating metabolites and the HCFP previously described were analysed using linear regression models. The analyses were adjusted for age, sex, BMI, fasting glucose, LDL cholesterol, high-density lipoprotein (HDL) cholesterol, triglycerides, systolic blood pressure, antihypertensive treatment, season for dietary data collection, alcohol intake and smoking status. In this initial analysis, associations were considered significant at a Bonferroni level $\mathrm{p}$ value limit of $<0.00044$. To test if the associations were independent of physical activity, sensitivity analysis was performed in the participants with complete data on physical activity $(n=2323)$.

To assess associations between the metabolites that were associated with the HCFP and specific food groups, a correlation matrix with Pearson's correlation coefficients was created. We compared the levels of metabolites with 10 selected food groups: fish and shellfish, fruits, vegetables, fermented milk, wholegrain portions, processed meat, non-processed read meat, root vegetables, leafy vegetables and sugar-sweetened beverages.

The five metabolites which associated significantly with the HCFP were in a first step related to the outcomes of incident CAD, type 2 diabetes, stroke, cardiovascular mortality and overall mortality using age-adjusted and sex-adjusted Cox proportional hazard models.

To visualise the relationships between the levels of healthy dietary biomarkers and morbidity and mortality, metabolite levels were divided into four quartiles and one Kaplan-Meier estimator per quartile was created.

Metabolites that were significant in the sex-adjusted and ageadjusted Cox regressions were further analysed in Cox regression models adjusted for age, sex, BMI, fasting glucose, LDL cholesterol, HDL cholesterol, triglycerides, systolic blood pressure, antihypertensive treatment, alcohol intake and smoking status. Schoenfeld residuals test was used to check the proportional hazard assumptions. To test if the Cox regression models were independent of physical activity, sensitivity analysis was performed in the participants with complete data on physical activity $(\mathrm{n}=2993)$.

Lastly, association between ergothioneine quartiles and risk factors for cardiometabolic disease were analysed.

\section{RESULTS}

\section{General characteristics of the study population}

The average baseline age of the participants was 57.4 years at baseline and $61 \%$ were female (table 1). Among the 3236 MDC-CC participants free from type 2 diabetes, $\mathrm{CAD}$ and stroke at the baseline, we identified 362 incident cases of type 2 diabetes, 316 of CAD and 271 of stroke during a median follow-up time of 21.4 years. During the same follow-up time, 843 participants died, of whom 229 died of cardiovascular causes.

\section{Identification of healthy dietary biomarkers}

Among the 112 measured metabolites, 5 showed significant positive associations with the HCFP and 1 had a significant negative association in the linear regression models adjusted for traditional cardiovascular risk factors (Bonferroni adjusted cut-off $\mathrm{p}<0.00044$ ) (figure 1 and online supplementary table 
Table 1 Study participants with complete data after exclusion of participants with prevalent diabetes mellitus, coronary artery disease and stroke

\begin{tabular}{|c|c|c|c|}
\hline & $\begin{array}{l}\text { Study participants } n=3236 \\
\text { mean } \pm \text { SD or } \%\end{array}$ & $\begin{array}{l}\text { Study participants with complete dietary } \\
\text { data } n=2513 \text { mean } \pm S D \text { or } \%\end{array}$ & $\begin{array}{l}\text { Study participants with complete data on } \\
\text { physical activity } n=2993 \text { mean } \pm \text { SD or } \%\end{array}$ \\
\hline Age (years) & $57.4 \pm 6.0$ & $57.4 \pm 6.0$ & $57.4 \pm 6.0$ \\
\hline Sex (\% female) & $61 \%$ & $60 \%$ & $61 \%$ \\
\hline Smokers & $27 \%$ & $28 \%$ & $27 \%$ \\
\hline BMI $\left(\mathrm{kg} / \mathrm{m}^{2}\right)$ & $25.3 \pm 3.7$ & $25.3 \pm 3.7$ & $25.3 \pm 3.7$ \\
\hline Systolic blood pressure (mm Hg) & $141 \pm 19$ & $141 \pm 19$ & $141 \pm 19$ \\
\hline Diastolic blood pressure $(\mathrm{mm} \mathrm{Hg})$ & $86.5 \pm 9.3$ & $86.4 \pm 9.3$ & $86.4 \pm 9.4$ \\
\hline Fasting glucose (mmol/L) & $4.90 \pm 0.43$ & $4.90 \pm 0.43$ & $4.90 \pm 0.44$ \\
\hline Fasting triglycerides (mmol/L) & $1.25 \pm 0.59$ & $1.23 \pm 0.57$ & $1.24 \pm 0.58$ \\
\hline Fasting HDL cholesterol (mmol/L) & $1.42 \pm 0.37$ & $1.42 \pm 0.37$ & $1.42 \pm 0.37$ \\
\hline Fasting LDL cholesterol (mmol/L) & $4.16 \pm 0.99$ & $4.13 \pm 0.96$ & $4.16 \pm 0.98$ \\
\hline Antihypertensive treatment & $13 \%$ & $11 \%$ & $13 \%$ \\
\hline Alcohol intake (g/day) & $10.1 \pm 11.7$ & $10.4 \pm 11.8$ & $10.2 \pm 11.7$ \\
\hline
\end{tabular}

Values are displayed as mean \pm SD or percentage.

BMI, body mass index; HDL, high-density lipoprotein; LDL, low-density lipoprotein.

2). Ergothioneine displayed the most significant association with the HCFP followed by proline betaine, acetylornithine and pantothenate. The metabolite that associated negatively with the
HCFP was urobilin. In sensitivity analyses, adjusting the linear regression models for physical activity did not change the associations (online supplementary table 3).

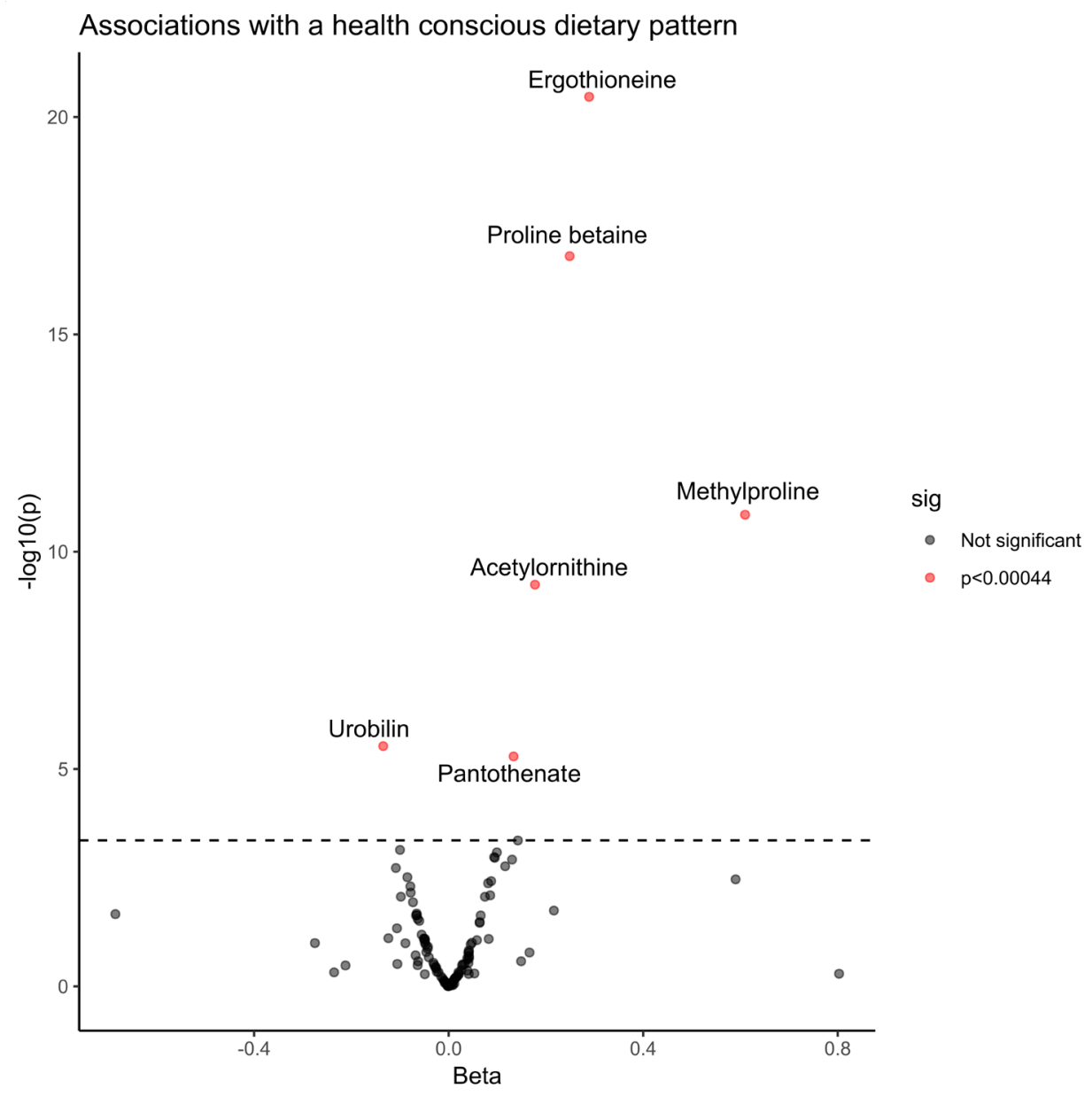

Figure 1 Identifying healthy dietary biomarkers using linear regression models. Five metabolites are found to be positively associated with the health conscious food pattern (HCFP) independent of traditional cardiovascular risk factors and one metabolite to be negatively associated. The linear regression models between the HCFP and fasting metabolite levels were adjusted for age, sex, body mass index, fasting glucose, fasting low-density lipoprotein cholesterol, fasting high-density lipoprotein cholesterol, fasting triglycerides, systolic blood pressure, antihypertensive treatment, season for dietary sampling, alcohol intake and smoking status. Beta represents a quintile increase or decrease in the five quintile scaled HCFP per SD increment of a metabolite. The cut-off for significance $(\mathrm{p}=0.00044)$ was calculated using Bonferroni correction, $\mathrm{n}=2513$. 


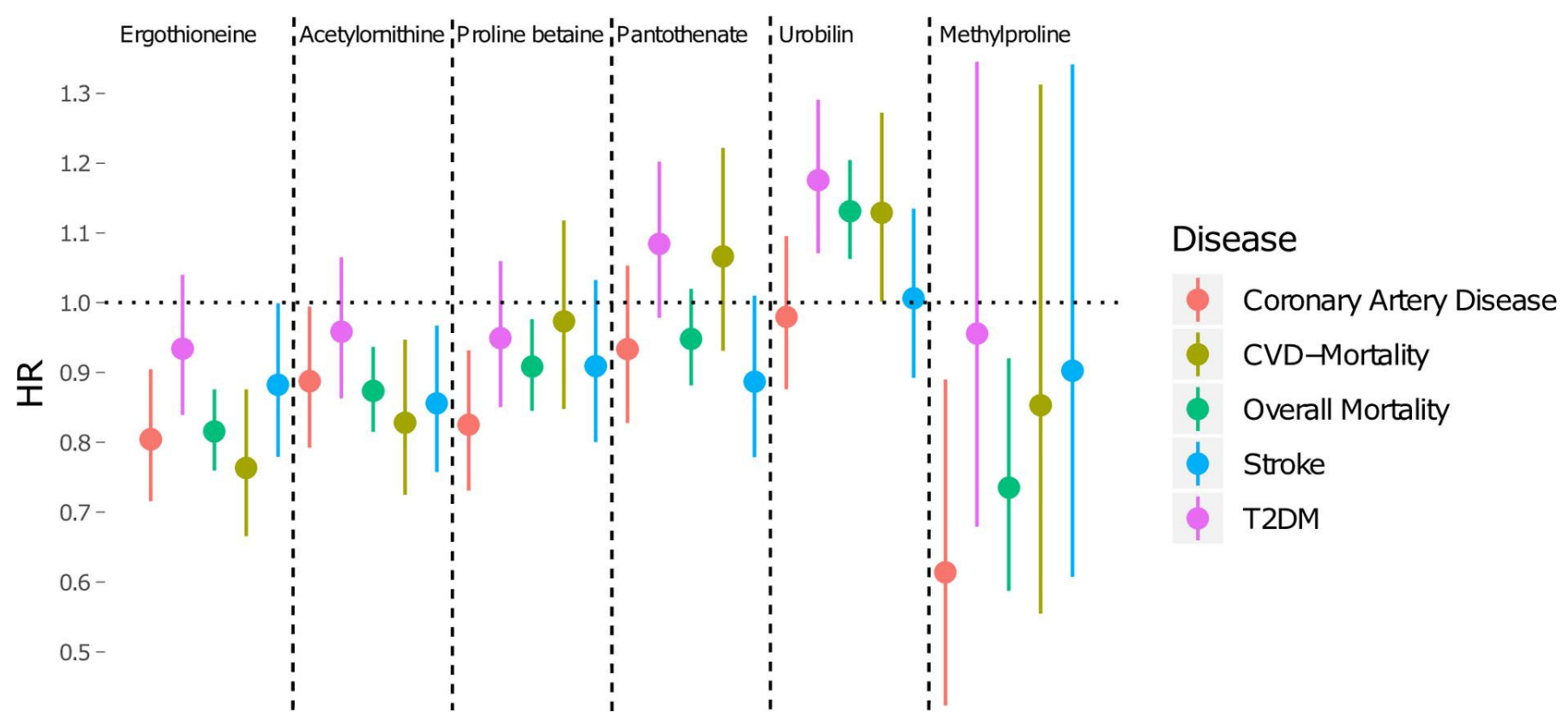

Figure 2 Sex-adjusted and age-adjusted Cox regressions. Cox proportional hazard models comparing the health conscious dietary biomarker levels with risk for all-cause and cause-specific mortality as well as for incident stroke, diabetes mellitus and coronary artery disease during the median follow-up time of 21.4 years. The models were adjusted for sex and age. The HR is calculated as the increase or decrease in risk per 1 SD increment of metabolite levels with $95 \% \mathrm{Cl}, \mathrm{n}=3236$. CVD, cardiovascular disease; T2DM, type 2 diabetes mellitus.

\section{Healthy dietary biomarkers and food groups}

All of the identified healthy dietary biomarkers as well as urobilin were significantly correlated with several food groups (online supplementary figure 1). The strongest correlation was observed between ergothioneine and vegetable intake, with a Pearson's correlation coefficient of $0.2(p=2 e-29)$.

\section{Dietary biomarkers and cardiovascular end points}

Next, we investigated association between plasma levels of the five healthy dietary biomarkers and of urobilin, and incidence of cardiometabolic disease as well as overall and cardiovascular mortality. Four healthy dietary biomarkers as well as urobilin were associated with at least one cardiometabolic disease end point in the Cox proportional hazards models adjusted for sex and age (figure 2). Ergothioneine was the metabolite most significantly associated with lower morbidity and mortality, being associated with a lower risk of CAD (HR per 1 SD increment of ergothioneine, $\mathrm{HR}=0.80,95 \% \mathrm{CI} 0.72$ to $0.90, \mathrm{p}=3 \mathrm{e}-$ $4)$, stroke $(\mathrm{HR}=0.86,95 \% \mathrm{CI} 0.76$ to $0.97, \mathrm{p}=0.01)$ death of all causes $(\mathrm{HR}=0.82,95 \% \mathrm{CI} 0.76$ to $0.88, \mathrm{p}=2 \mathrm{e}-8)$ and death of cardiovascular causes $(\mathrm{HR}=0.76,95 \%$ CI 0.67 to $0.88, \mathrm{p}=7 \mathrm{e}-$ 4). Kaplan-Meier curves are found in the online supplementary figures 2-7.

\section{Ergothioneine independently predicts cardiometabolic disease}

To test if the associations between metabolite levels and end points were independent from traditional cardiometabolic risk factors (age, sex, BMI, fasting glucose, LDL cholesterol, HDL cholesterol, triglycerides, systolic blood pressure, antihypertensive treatment, alcohol intake and smoking status), multivariable Cox regression analyses were performed. Five metabolites were significantly associated with at least one cardiometabolic end point in the fully adjusted models (figure 3 and supplementary table 4). Ergothioneine remained significantly associated with a lower risk of CAD, cardiovascular mortality and overall mortality. Acetylornithine remained significantly associated with a lower risk for cardiovascular mortality, overall mortality and stroke. Proline betaine was associated with a lower risk for CAD and overall mortality. Methylproline was significantly associated with a lower risk for overall mortality. Urobilin remained significantly associated with an increased risk of overall mortality and type 2 diabetes. The proportional hazard assumptions were met for all models except the model with urobilin and overall mortality (online supplementary table 4).

In sensitivity analyses, adjusting the Cox regression models for physical activity did not affect the HRs (online supplementary table 5).

\section{Metabolite levels and cardiovascular risk factors}

The associations between traditional cardiovascular risk factors and ergothioneine were overall weak, except for alcohol intake, which was strongly associated with ergothioneine levels, and for HDL cholesterol, which was negatively associated (online supplementary table 6).

\section{DISCUSSION}

\section{Main findings}

In this population-based prospective study we identify plasma metabolites that, for the first time, tie together an HCFP and a lower risk of cardiometabolic morbidity and mortality during a long-term follow-up. In particular, increased level of the amino acid ergothioneine was strongly and independently associated with both HCFP and a lower risk of future CAD and cardiovascular and all-cause mortality. Overall, our findings suggest novel pathways linking diet to cardiometabolic health.

\section{Healthy dietary biomarkers}

Several of the metabolites we found to be associated with the HCFP have previously been correlated with self-reported intake of specific food groups or items. Ergothioneine exists in many 


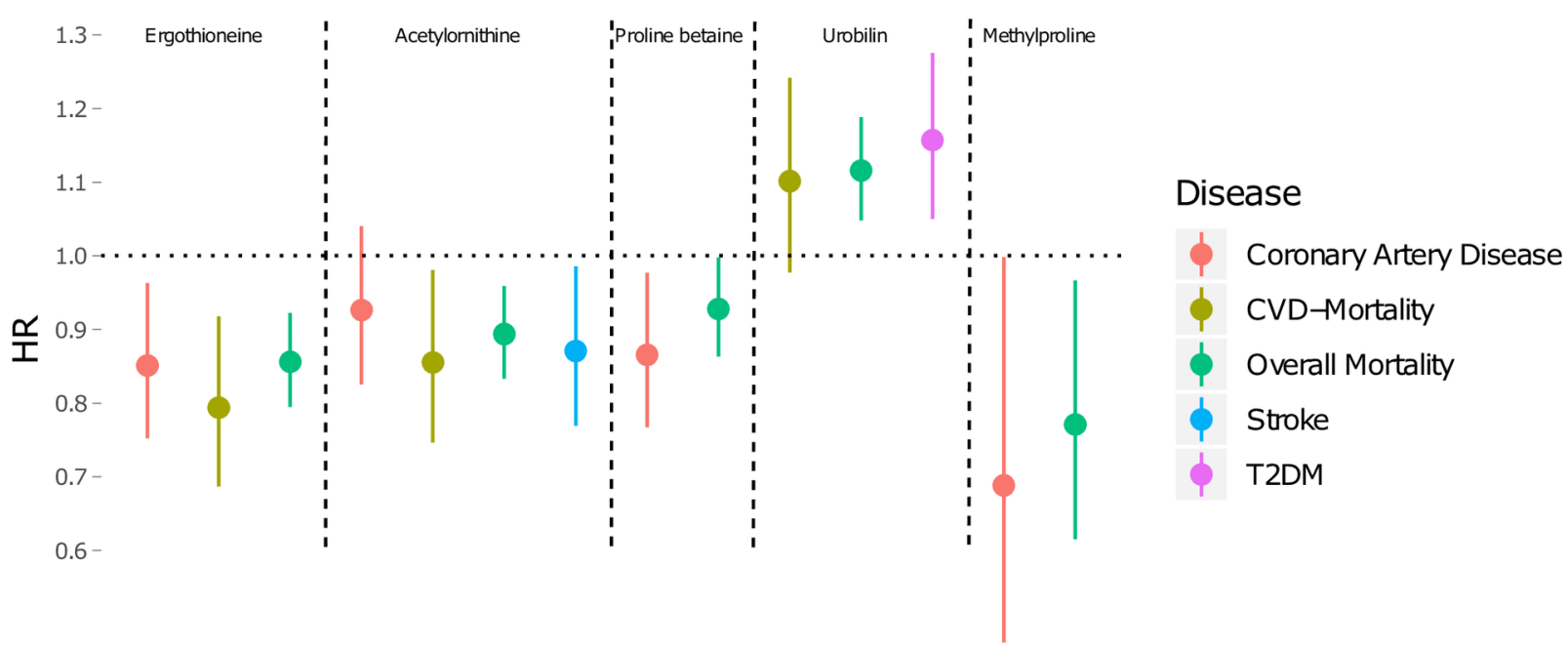

Figure 3 Fully adjusted Cox regressions. Cox proportional hazard models comparing the health conscious dietary biomarker levels with risk for overall and cardiovascular mortality as well as for incident stroke, diabetes mellitus and coronary artery disease during the median follow-up time of 21.4 years. The models were adjusted for age, sex, body mass index, fasting glucose, fasting low-density lipoprotein cholesterol, fasting highdensity lipoprotein cholesterol, fasting triglycerides, systolic blood pressure, antihypertensive treatment, alcohol intake and smoking status. The HR is calculated as the increase or decrease in risk per 1 SD increment of metabolite levels with $95 \% \mathrm{Cl}, \mathrm{n}=3236$. CVD, cardiovascular disease; T2DM, type 2 diabetes mellitus.

dietary sources ${ }^{17}$ and has especially high levels in mushrooms, tempeh and garlic. ${ }^{18}$ Ergothioneine has previously been associated with a higher intake of vegetables, seafood and with a lower intake of solid fats and added sugar as well as associated with healthy food patterns, ${ }^{19}$ which is in line with our results concerning the association with intake of vegetables, seafood and the HCFP. Proline betaine, also known as stachydrine, and methylproline are both known biomarkers for citrus fruit intake which could explain the association with fruit intake in our study. ${ }^{20}$ Acetylornithine has been associated with a higher intake of vegetables which was also confirmed in our study. ${ }^{19}$ Pantothenate, also known as vitamin $\mathrm{B}_{5}$, is widely distributed in all food groups. ${ }^{21}$ In contrast, urobilin, which in our study displayed a negative association with the HCFP, has not previously been associated with any dietary intake. The correlation between metabolite levels and food groups was modest, but the correlation coefficients between ergothioneine and food groups were similar to previously reported values. ${ }^{22}$ Even though the MDC uses an extensive dietary sampling method, measurement inaccuracy is likely to attenuate the observed correlations. ${ }^{23}$

\section{Ergothioneine: a biomarker of both healthy diet and low cardiometabolic risk}

Ergothioneine had the strongest association with the HCFP and also the most evident protective associations with cardiometabolic morbidity and mortality independent of traditional risk factors. Our results thus suggest ergothioneine as a biomarker of both healthy dietary intake and of low risk of future cardiometabolic disease. Ergothioneine has earlier been shown to protect rodents from ischaemic reperfusion injury ${ }^{24}$ and has also previously suggested to be an anti-oxidant with potential beneficial effects on the human body. Ergothioneine differs from other suggested anti-oxidants by having a specific transporter that has been suggested to be upregulated in areas of inflammation, providing ergothioneine a potential for a more controlled antioxidant function. ${ }^{18}$ Having higher levels of ergothioneine could in a reactionary manner protect from oxidative stress, which is thought to be an important factor in the pathogenesis of $\mathrm{CVD}^{25}$ and could explain our findings.
A recent study conducted in healthy humans showed that oral administration of ergothioneine increased the levels of circulating ergothioneine as well as decreased levels of some biomarkers of oxidative damage. ${ }^{26}$ Intervention trials with randomised treatment regimen design, are required to investigate if this potential anti-oxidant effect can decrease the risk of cardiometabolic disease.

The positive correlation between ergothioneine and alcohol intake has previously been demonstrated, ${ }^{22}$ an association that can either be explained by ergothioneine being present in alcoholic beverages, ${ }^{17}$ or by alcohol changing the absorption efficiency of ergothioneine present in other dietary sources.

\section{Other dietary biomarkers and cardiometabolic disease}

In our study, proline betaine showed an association with a decreased risk for CAD and overall mortality but the latter association vanished after adjustment for traditional risk factors. In support for a role of proline betaine in CAD protection, a previous study showed that addition of proline betaine to endothelial cells cultured in glucotoxic conditions ameliorated the detrimental effect of high glucose on the endothelial cells. ${ }^{27}$ The association could also be explained by proline betaine being a confounding variable for another compound; proline betaine is a known biomarker for citrus fruit, which contains many other metabolites not measured using our method. ${ }^{20}$

To our knowledge, the observed associations between decreased risk of cardiometabolic end points and the healthy dietary biomarkers acetylornithine and methylproline represent novel findings. Acetylornithine was the only metabolite associated with a lower risk for stroke in the fully adjusted models, further studies are needed to investigate the potential mechanism linking high acetylornithine levels to better cardiometabolic health. Urobilin has to our knowledge not previously been associated with an increased risk of type 2 diabetes or mortality, but recently associated with an increased risk of heart failure. ${ }^{28}$ More research is needed to explain the association between haemoglobin breakdown components and CVD. 


\section{Key messages}

\section{What is already known on this subject?}

- Dietary intake plays a great role in the development of cardiometabolic disease.

- Dietary intake is known to correlate with certain metabolites.

\section{What might this study add?}

- We identify biomarkers associated independently from traditional risk factors with a healthy food pattern and incidence of cardiometabolic morbidity and mortality.

- The previously suggested anti-oxidant ergothioneine was associated with a reduced risk of cardiometabolic disease and mortality.

\section{How might this impact on clinical practice?}

- Our results encourages controlled specific dietary intervention studies, and ergothioneine supplementation, aimed at testing if raising ergothioneine plasma concentration may reduce risk of cardiometabolic disease.

\section{Strengths and limitations}

The large and prospective population-based cohort design, with $>20$ years of follow-up and validated end points, comprise major strengths of our study. Moreover, we benefit from high-quality dietary data from the thorough MDC diet assessment method. ${ }^{15}$

As the dietary intakes, cardiometabolic risk factors and the metabolomics data were only collected at baseline of the study, changes in these factors cannot be studied or adjusted for. However, the study participants in MDC were middle-aged or older at baseline and have most likely in general rather established dietary habits less prone to radical changes.

As in all observational studies, it is not possible to prove that the observed associations between the healthy dietary biomarkers and end points are casual. However, by using a prospective study design and models fully adjusted for traditional risk factors, we think that our results provide strong base for future intervention studies examining the observed diet-metabolite-disease associations. Moreover, the healthy diet-associated metabolites could be useful biomarkers of diet adherence.

\section{CONCLUSION}

We identify biomarkers associated independently from traditional risk factors with a healthy food pattern and incidence of cardiometabolic morbidity and mortality. The metabolite ergothioneine had the most significant correlation to the healthy food pattern and was the most predictive for low risk of CVD. Our results encourage controlled specific dietary intervention studies, and ergothioneine supplementation, aimed at testing if raising ergothioneine plasma concentration may reduce risk of cardiometabolic disease.

\footnotetext{
Acknowledgements The authors would like to thank the staff and participants of the Malmö Diet and Cancer Study.

Contributors ES contributed to study design, acquisition and interpretation of data, statistical analyses and drafted the manuscript. UE, SH and FO contributed to collection and interpretation of data and statistical analysis. MO-M assisted in data interpretation. CF and OM contributed to study concept and design, interpretation of data and statistical analyses. All authors made intellectual contributions to drafting and/or revising the manuscript and approved the final version. ES, CF, FO and OM had full access to the data. The manuscript is original research, has not been published and is not submitted to be considered for publication elsewhere, in whole or in part, in any language. There are no potentially overlapping papers in
}

preparation, submission or published. The authors declare that there is no duality of interest associated with this manuscript.

Funding This study was funded by Crafoordska Stiftelsen, Vetenskapsrådet, Novo Nordisk, Åke Wiberg Stiftelse, Göran Gustafssons Stiftelse för Naturvetenskaplig och Medicinsk Forskning, Knut och Alice Wallenbergs Stiftelse, Kungliga Fysiografiska Sällskapet i Lund, the Ernhold Lundström Research Foundation, Region Skåne, Skåne University Hospital, European Foundation for the Study of Diabetes (grant no: EFSD 2015/338), Hjärt-Lungfonden, H2020 European Research Council, Diabetesfonden, Direktör Albert Påhlssons Stiftelse. OM was supported by Knut and Alice Wallenberg Foundation, Göran Gustafsson Foundation, the Swedish Heart and Lung Foundation, the Swedish Research Council, the Novo Nordisk Foundation, Region Skåne, Skåne University Hospital. CF was supported by the Albert Påhlsson Research Foundation, the Crafoord Research Foundation, the Ernhold Lundström Research Foundation, the Royal Physiographic Society of Lund and the Åke Wiberg Foundation. MO-M was supported by the European Research Council (Consolidator grant nr 649021, Orho-Melander), the Swedish Research Council, the Swedish Heart and Lung Foundation, the Novo Nordic Foundation, the Swedish Diabetes Foundation, the European Foundation for Study of Diabetes (EFSD 2015/338) and the Region Skåne, Skåne University Hospital. FO was supported by the Ernhold Lundström Research Foundation.

Competing interests None declared.

Patient consent for publication Not required.

Ethics approval The study was approved by the Ethics Committee of Lund University, Lund, Sweden (LU 51-90).

Provenance and peer review Not commissioned; externally peer reviewed. Data availability statement Data are available upon reasonable request.

Open access This is an open access article distributed in accordance with the Creative Commons Attribution Non Commercial (CC BY-NC 4.0) license, which permits others to distribute, remix, adapt, build upon this work non-commercially, and license their derivative works on different terms, provided the original work is properly cited, appropriate credit is given, any changes made indicated, and the use is non-commercial. See: http://creativecommons.org/licenses/by-nc/4.0/.

\section{ORCID iD}

Einar Smith http://orcid.org/0000-0002-4150-2459

\section{REFERENCES}

1 Estruch R, Ros E, Salas-Salvadó J, et al. Primary prevention of cardiovascular disease with a Mediterranean diet supplemented with extra-virgin olive oil or nuts. $N$ Eng/ J Med 2018;378:e34

2 Salas-Salvadó J, Martinez-González M.Á., Bulló M, et al. The role of diet in the prevention of type 2 diabetes. Nutrition, Metabolism and Cardiovascular Diseases 2011;21:B32-48.

3 Clar C, Al-Khudairy L, Loveman E, et al. Low glycaemic index diets for the prevention of cardiovascular disease. Cochrane Database of Systematic Reviews 2017;26.

4 Rodríguez-Monforte M, Flores-Mateo G, Sánchez E. Dietary patterns and CVD: a systematic review and meta-analysis of observational studies. Br J Nutr 2015;114:1341-59.

5 Scalbert A, Brennan L, Manach C, et al. The food metabolome: a window over dietary exposure. Am J Clin Nutr 2014;99:1286-308.

6 Rebholz CM, Lichtenstein AH, Zheng Z, et al. Serum untargeted metabolomic profile of the dietary approaches to stop hypertension (DASH) dietary pattern. Am J Clin Nutr 2018.

7 Hanhineva K, Lankinen MA, Pedret A, et al. Nontargeted metabolite profiling discriminates diet-specific biomarkers for consumption of whole grains, fatty fish, and bilberries in a randomized controlled trial. J Nutr 2015;145:7-17.

8 Wang TJ, Larson MG, Vasan RS, et al. Metabolite profiles and the risk of developing diabetes. Nat Med 2011;17:448-53.

9 Ottosson F, Smith E, Melander 0, et al. Altered asparagine and glutamate homeostasis precede coronary artery disease and type-2 diabetes. J Clin Endocrinol Metab 2018.

10 Cheng S, Larson MG, McCabe EL, et al. Distinct metabolomic signatures are associated with longevity in humans. Nat Commun 2015;6:6791.

11 Guertin KA, Moore SC, Sampson JN, et al. Metabolomics in nutritional epidemiology: identifying metabolites associated with diet and quantifying their potential to uncover diet-disease relations in populations. Am J Clin Nutr 2014;100:208-17.

12 Ericson U, Brunkwall L, Alves Dias J, et al. Food patterns in relation to weight change and incidence of type 2 diabetes, coronary events and stroke in the Malmo diet and cancer cohort. Eur J Nutr 2018.

13 Berglund G, Elmstahl S, Janzon L, et al. The Malmo diet and cancer study. design and feasibility. J Intern Med 1993;233:45-51.

14 Persson M, Hedblad B, Nelson JJ, et al. Elevated Lp-PLA 2 Levels Add Prognostic Information to the Metabolic Syndrome on Incidence of Cardiovascular Events Among Middle-Aged Nondiabetic Subjects. Arterioscler Thromb Vasc Biol 2007;27:1411-6. 
15 Wirfält E, Mattisson I, Johansson U, et al. A methodological report from the Malmö diet and cancer study: development and evaluation of altered routines in dietary data processing. Nutr J 2002;1:3.

16 Ottosson F, Ericson U, Almgren P, et al. Postprandial levels of branch Chained and aromatic amino acids associate with fasting glycaemia. J Amino Acids 2016;2016:1-9.

17 Ey J, Schömig E, Taubert D. Dietary sources and antioxidant effects of ergothioneine. J Agric Food Chem 2007;55:6466-74.

18 Halliwell B, Cheah IK, Tang RMY. Ergothioneine - a diet-derived antioxidant with therapeutic potential. FEBS Lett 2018;592:3357-66.

19 Playdon MC, Moore SC, Derkach A, et al. Identifying biomarkers of dietary patterns by using metabolomics. Am J Clin Nutr 2017;105:450-65.

20 Playdon MC, Sampson JN, Cross AJ, et al. Comparing metabolite profiles of habitual diet in serum and urine. Am J Clin Nutr 2016;104:776-89.

21 Strain J, Hughes C, Pentieva K, et al. The B-Vitamins. In: Biesalski HK, Drewnowski A, Dwyer JT, et al, eds. Sustainable nutrition in a changing world. Cham: Springer International Publishing, 2017: 197.

22 Playdon MC, Ziegler RG, Sampson JN, et al. Nutritional metabolomics and breast cancer risk in a prospective study. Am J Clin Nutr 2017;106:637-49.
23 Freedman LS, Schatzkin A, Midthune $D$, et al. Dealing with dietary measurement error in nutritional cohort studies. J Natl Cancer Inst 2011;103:1086-92.

24 Sakrak 0, Kerem M, Bedirli A, et al. Ergothioneine modulates proinflammatory cytokines and heat shock protein 70 in mesenteric ischemia and reperfusion injury. Journal of Surgical Research 2008;144:36-42.

25 Higashi Y, Noma K, Yoshizumi M, et al. Endothelial function and oxidative stress in cardiovascular diseases. Circ J 2009;73:411-8.

26 Cheah IK, Tang RMY, Yew TSZ, et al. Administration of pure ergothioneine to healthy human subjects: uptake, metabolism, and effects on biomarkers of oxidative damage and inflammation. Antioxid Redox Signal 2017;26:193-206.

27 Servillo L, D'Onofrio N, Longobardi L, et al. Stachydrine ameliorates high-glucose induced endothelial cell senescence and SIRT1 downregulation. J Cell Biochem 2013;114:2522-30.

28 Stenemo M, Ganna A, Salihovic S, et al. The metabolites urobilin and sphingomyelin $(30: 1)$ are associated with incident heart failure in the general population. ESC Heart Failure 2019;6:764-73. 